Elias Zintzaras · Ioannis Stefanidis

\title{
Association between the GLUT1 gene polymorphism and the risk of diabetic nephropathy: a meta-analysis
}

Received: 7 September 2004 / Accepted: 29 November 2004 / Published online: 29 January 2005

(C) The Japan Society of Human Genetics and Springer-Verlag 2005

\begin{abstract}
The association between diabetic nephropathy (DN) and the $X b \alpha \mathrm{I}$ polymorphism in the GLUT1 gene has been investigated in several case-control studies. These studies rendered contradictory results: the allele $X b \alpha \mathrm{I}(-)$ was shown either to be a risk factor or neutral, or even protective for the development of the disease. To shed some light on these inconclusive findings, a metaanalysis of all available studies relating the $X b \alpha \mathrm{I}$ polymorphism to the risk of developing DN was conducted. Five out of six identified studies included Caucasian populations, and only one involved samples from an Asian population. Overall, the meta-analysis suggested large heterogeneity between studies $\left(P<0.01, I^{2}=68 \%\right)$ and lack of association between allele $X b \alpha \mathrm{I}(-)$ and the risk of developing DN relative to allele $X b \alpha \mathrm{I}(+)$ : random effects odds ratio $(\mathrm{OR})=1.26[95 \% \mathrm{CI}(0.93,1.69)]$. Excluding one study with the controls not in HardyWeinberg equilibrium, the sensitivity analysis revealed that heterogeneity $\left(P=0.28, I^{2}=21 \%\right)$ could be explained, and then, there is an overall association: fixed effects $\mathrm{OR}=1.34[95 \% \mathrm{CI}(1.13,1.60)]$. Then, significant ORs were also found on analysis of subgroups: for the Caucasian population, fixed effects $\mathrm{OR}=1.29[95 \% \mathrm{CI}$ $(1.08,1.56)]$ and for the type 2 diabetic patients fixed effects $\mathrm{OR}=1.69[95 \%$ CI $(1.09,2.63)]$. In type 1 diabetes, there is a moderate heterogeneity $(P=0.19$, $\left.I^{2}=41 \%\right)$ with fixed effects $\mathrm{OR}=1.29[95 \%$ CI $(1.06$, 1.56)] and random effects $\mathrm{OR}=1.32[95 \%$ CI $(1.01$, 1.71)]. There is a source of bias in the selected studies: large studies failed to show association while small
\end{abstract}

E. Zintzaras $(\bowtie)$

Department of Biomathematics,

University of Thessaly School of Medicine,

Papakyriazy 22, Larissa, 41222, Greece

E-mail: zintza@med.uth.gr

Tel.: + 30-2410-565270

Fax: $+30-2410-565270$

I. Stefanidis

Division of Nephrology, University Hospital of Larissa,

University of Thessaly School of Medicine,

Papakyriazy 22, Larissa, 41222, Greece studies claimed an association. Although there is evidence of association between GLUT1 and DN, the above findings reinforce the need for further and more rigorous association studies.

Keywords Diabetic nephropathy · GLUT1 · Polymorphism $\cdot$ Meta-analysis · Diabetes

\section{Introduction}

Diabetic nephropathy is a serious, chronic microangiopathic complication of both types 1 and 2 diabetes and has become the most frequent cause of end-stage renal disease in recent years (Valderrábano et al. 1996). In the early 1990s, it was convincingly proved that the single most important factor in the development of this nephropathy is hyperglycemia (The Diabetes Control and Complications Trial Research Group 1993). However, there is strong evidence for the involvement of genetic factors in its pathophysiology. Not all diabetic patients develop diabetic nephropathy, indicating specific genetically defined predisposing factors. Furthermore, significant familiar clustering of diabetic nephropathy has been shown in both types of diabetes (1 and 2) and in different populations (Quinn et al. 1996; Strojek et al. 1997).

In previous studies, it has been postulated that the $X b \alpha \mathrm{I}$ polymorphism in the glucose GLUT1 gene is involved in the development of diabetic nephropathy in patients with types 1 and 2 diabetes mellitus (Gutierrez et al. 1998; Liu et al. 1999; Tarnow et al. 2001; Hodgkinson et al. 2001; Grzeszczak et al. 2001; $\mathrm{Ng}$ et al. 2002). The $X b \alpha \mathrm{I}$ restriction fragment length polymorphism (RFLP) is located in the second intron of the GLUT1 gene approximately $4.5 \mathrm{kbp}$ upstream of exon 3 . The $X b \alpha \mathrm{I}$ RFLP consists of a guanine $(\mathrm{G})$ being transversed to a thymine (T), which abolishes the $X b \alpha \mathrm{I}$ recognition site. That gives rise to $X b \alpha \mathrm{I}(-)$ and $X b \alpha \mathrm{I}(+)$ alleles, which correspond to an $1.1 \mathrm{~kb}$ band and to a set of 0.9 and $0.2 \mathrm{~kb}$ bands, respectively. 
A number of case-control studies have investigated the association between the diabetes-related complications, such as nephropathy, and the $X b \alpha$ I polymorphism in the GLUT1 gene, but their results were inconclusive. Some studies urge that $X b \alpha \mathrm{I}(-)$ allele of the GLUT1 gene [designated (1.1)] relative to the $X b \alpha \mathrm{I}(+)$ allele [designated as (0.9)] is a risk factor for developing diabetic nephropathy (Liu et al. 1999; Hodgkinson et al. 2001; $\mathrm{Ng}$ et al. 2002), other investigations report no genetic association (Gutierrez et al. 1998; Tarnow et al. 2001), and other studies urge that allele (1.1) protects against diabetic nephropathy (Grzeszczak et al. 2001). However, in single studies, the significant associations between (1.1) allele and diabetic nephropathy are rather marginal and controversial.

To provide an answer to these contradictory results, a meta-analysis (Lau et al. 1997; Ioannidis 1998; Ntais et al. 2003a, 2003b) of all available studies relating the $X b \alpha \mathrm{I}$ polymorphism to the risk of developing diabetic nephropathy was conducted. In the meta-analysis, the estimates of the genetic association of each individual study and a pooled estimate of this association was obtained. In addition, the heterogeneity between studies was investigated.

\section{Materials and methods}

Selection of studies

All studies that investigate the association of the $X b \alpha \mathrm{I}$ polymorphism in the GLUT1 gene with the development of diabetic nephropathy published before May 2004 were considered in the meta-analysis. The studies were identified by extended computer-based searches of the PubMed database. As a search criterion, we used the following: "GLUT1" and ["diabetic nephropathy" or ("diabetes" and "nephropathy")]. The retrieved studies were then read in their entirety in order to assess their appropriateness for inclusion. All references cited in the studies were also reviewed to identify additional published work not indexed by the PubMed database. Abstracts, case reports, editorials, and review articles were excluded. The search was restricted to articles in English. Case-control studies that determined the distribution of the $X b \alpha \mathrm{I}$ genotypes in cases with diabetic nephropathy and in a control group were eligible for inclusion. A case was considered as diabetic nephropathy only when proteinuria and/or chronic renal failure (CRF) were/was detected. On the contrary, patients with microalbuminuria (i.e., urinary albumin excretion $30-300 \mathrm{mg} / 24 \mathrm{~h}$ ) were excluded. Although the presence of microalbuminuria may be an early finding in diabetic nephropathy, it is not invariably equivalent to it. The control group consisted of subjects with diabetes and free of diabetic nephropathy, i.e., without albuminuria (albumin excretion $<20 \mathrm{mg} / 24 \mathrm{~h}$, Table 1 ).

The distribution of the genotypes in the control group was tested to determine if it was in Hardy-Weinberg

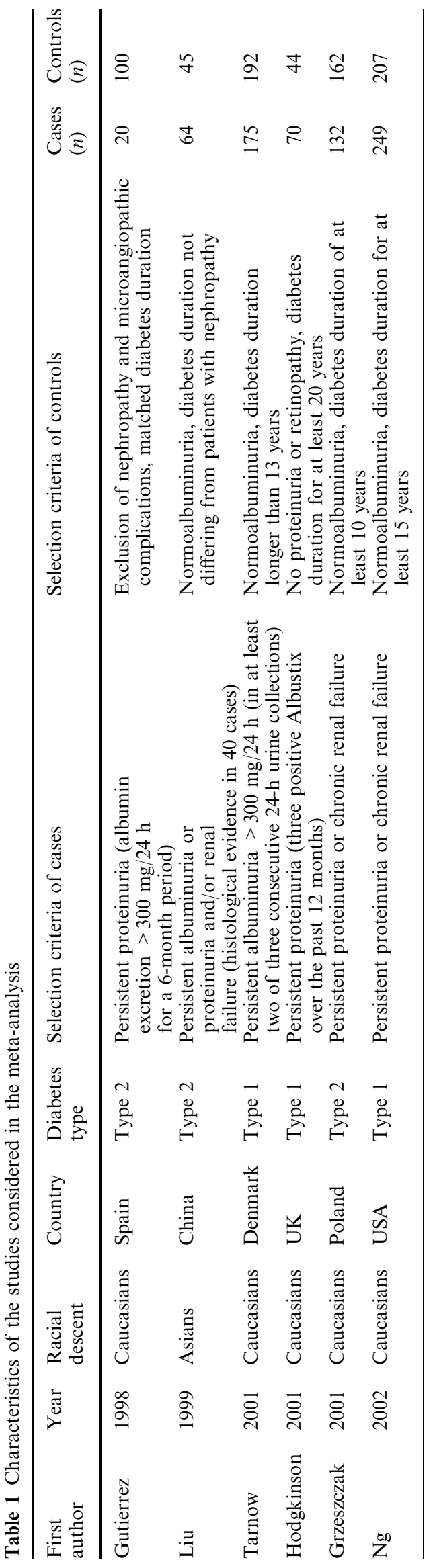


equilibrium $(P \geq 0.05)$ (Weir 1996, S.A.G.E. 2003). Studies with controls not in Hardy-Weinberg equilibrium were subjected to a sensitivity analysis. Studies based on pedigree data were excluded since they investigate linkage (Zintzaras and Ioannidis 2004) and not association.

\section{Data extraction}

From each study, the following information was extracted: first author, journal, year of publication, racial descent of study population, demographics, matching, validity of the genotyping method, and the number of cases and controls for each $X b \alpha \mathrm{I}$ genotype. The frequencies of the alleles were calculated for cases and controls from the corresponding genotype distributions. In addition, it was recorded whether the genotyping in each study was blinded to clinical status.

\section{Meta-analysis}

Prior to the main analysis, the significance of the association between the two alleles of $X b \alpha \mathrm{I}$ and risk of having diabetic nephropathy was evaluated for each study, separately. The main analysis examined the overall association of (1.1) allele with the risk of diabetic nephropathy relative to the (0.9) allele. The contrast of homozygotes $(1.1) /(1.1)$ versus $(0.9) /(0.9)$, the contrasts $(0.9) /(1.1)+(1.1) /(1.1)$ versus $(0.9) /(0.9)$, and $(1.1) /(1.1)$ versus $(0.9) /(1.1)+(0.9) /(0.9)$ were also examined. All associations were indicated as odds ratios (ORs) with the corresponding $95 \%$ confidence interval $(\mathrm{CI})$. Based on the individual ORs, a pooled OR was estimated.

Heterogeneity between studies was tested using the $Q$ statistic, which is a weighted sum of squares of the deviations of individual study OR estimates from the overall estimate (Cochran 1954). When the ORs are homogeneous, $Q$ follows a chi-squared distribution with $r-1$ ( $\mathrm{r}$ is the number of studies) degrees of freedom (d.f.). If $P<0.10$, then heterogeneity was considered statistically significant. Heterogeneity was quantified with the $I^{2}$ metric $\left(I^{2}=(Q-\right.$ d.f. $\left.) / Q\right)$, which is independent of the number of studies in the meta-analysis (Higgins et al. 2003). $I^{2}$ takes values between 0 and $100 \%$ with higher values denoting greater degree of heterogeneity $\left(I^{2}=0-25 \%\right.$ : no heterogeneity; $I^{2}=25-$ $50 \%$ : moderate heterogeneity; $I^{2}=50-75 \%$ : large heterogeneity; $I^{2}=75-100 \%$ : extreme heterogeneity).

The pooled OR was estimated using fixed effects (Mantel-Hasnszel) and random effects (DerSimonian and Laird) models. Random effects modelling assumes a genuine diversity in the results of various studies, and it incorporates to the calculations a between study variance. Therefore, when there is heterogeneity between studies, then the pooled OR is estimated using the random effects model (Whitehead 2002). Adjusted estimates of OR were considered whenever possible in a separate analysis. A cumulative meta-analysis (Lau et al. 1992; Whitehead 1997) and recursive meta-analysis were carried out in order to evaluate the trend of pooled OR for the allele contrast [(1.1) versus (0.9)] in time. The publication bias was tested for the allele contrast based on the linear regression model $y_{i}=\alpha+\beta \quad x_{i}$ where the dependent variable $y_{i}$ is the standardized estimate of $\theta_{i}\left(y_{i}=\theta_{i} \sqrt{w_{i}}\right)$ and the independent variable $x_{i}$ is the precision $\left(x_{i}=\sqrt{w_{i}}\right)$. A test of publication bias would be a test of the null hypothesis that $\alpha$ is equal to zero $(\alpha$-statistic), i.e., $\alpha$ provides a measure of funnel plot asymmetry (Egger et al. 1997; Whitehead 2002; Ioannidis et al. 2003).

The main analysis included all available data. Subgroup analyses for the studies of the Caucasian population and for each type of diabetes were also performed. In sensitivity analyses, the effect of excluding specific studies was examined. Analyses were performed using Meta-Analyst (Joseph Lau, Boston, MA, USA, 1998) and SAS routines provided by the Medical and Pharmaceutics Statistics Research Unit, University of Reading, UK (Whitehead 2002).

\section{Results}

\section{Eligible studies}

The literature review identified 17 titles that met the search criteria. After review, 14 titles were judged to be potentially relevant. The abstracts of these articles were further reviewed, and full articles of selected studies were read to assess their appropriateness for meta-analysis. Six studies investigating the association between the GLUT1 gene $X b \alpha \mathrm{I}$ polymorphism and diabetic nephropathy met the inclusion criteria (Table 1). These studies were published between 1998 and 2002. Three studies dealt with type 1 diabetes and three with type 2 diabetes. In five studies (Gutierrez et al. 1998; Tarnow et al. 2001; Hodgkinson et al. 2001; Grzeszczak et al. 2001; Ng et al. 2002), the subjects were Caucasians and in one, the subjects were Asians (Liu et al. 1999). In all studies, the cases (patients with diabetes and nephropathy) were well defined following similar inclusion criteria although the data sources were different: in one study ( $\mathrm{Ng}$ et al. 2002), the data originated from a database; the other studies were cohorts. The controls were diabetic patients free of nephropathy (Table 1). In one study (Grzeszczak et al. 2001), the association reported was based on a comparison of cases (diabetic nephropathy) both to diabetic patients without nephropathy and to healthy controls. However, in agreement to the study selection criteria, only diabetic patients without nephropathy (normoalbuminuria) from this study were considered as controls for the metaanalysis.

In one study (Tarnow et al. 2001), it was denoted that there was matching for gender, age, and diabetes duration. In three studies (Hodgkinson et al. 2001; 
Grzeszczak et al. 2001; Ng et al. 2002), controls were defined as all patients without signs of diabetic nephropathy and a duration of diabetes longer than 10 years. In the control group, the diabetes duration varied from 12 to 31 years. Age was specified only into two studies (Tarnow et al. 2001; Grzeszczak et al. 2001). In all studies, gender was evenly distributed, and all used validated genotyping method: PCR and restriction of the PCR product with the $X b \alpha \mathrm{I}$ enzyme.

\section{Summary statistics}

In total, the studies included 710 cases with diabetic nephropathy and 750 control diabetic patients without nephropathy. In the diabetic nephropathy group, allele (0.9) was the most frequent: $62 \%$ [95\% CI $(59,65 \%)$ ] whereas the frequency of the allele (1.1) was 38\% [95\% CI $(36,41 \%)]$. In the control group, the prevalence of allele (0.9) was $66 \%$ [95\% CI $(64,68 \%)]$, and the prevalence of allele (1.1) was $34 \%$ [95\% CI $(32,36 \%)]$. The prevalence of homozygotes $(0.9) /(0.9)$ among patients with diabetic nephropathy and controls were $38 \%$ [95\% CI $(34,42 \%)]$ and $43 \%$ [95\% CI $(39,46 \%)]$, respectively. The prevalence of (1.1)/(1.1) among patients with diabetic nephropathy and the controls were $14 \%$ [95\% CI $(12,17 \%)]$ and $11 \%$ [95\% CI $(9,13 \%)]$, respectively. The prevalence of heterozygotes $(0.9) /(0.1)$ among patients with diabetic nephropathy and controls were $49 \%$ [95\% CI $(45,53 \%)]$ and $46 \%$ [95\% CI $(42$, $50 \%$ )], respectively (Table 2).

In one study (Grzeszczak et al. 2001), distribution of the genotypes in control groups was not in HardyWeinberg equilibrium $(P<0.05)$, indicating genotyping errors and population stratification (Silverman et al. 2000; Xu et al. 2002; de Jong et al. 2003). Therefore, a sensitivity analysis was carried out for this study, and the results should be interpreted with caution.

Main results, subgroup, and sensitivity analyses

The main analysis for investigating the association of the allele (1.1) and the risk of developing diabetic nephropathy relative to the allele $(0.9)$ showed that there was heterogeneity $\left(P<0.01, I^{2}=68 \%\right)$ between the six study comparisons (Gutierrez et al. 1998; Liu et al. 1999; Tarnow et al. 2001; Hodgkinson et al. 2001; Grzeszczak et al. 2001; $\mathrm{Ng}$ et al. 2002), and the random effects pooled OR was not significant $\mathrm{OR}=1.26[95 \% \mathrm{CI}(0.93$, 1.69)] (Table 3, Fig. 1).

In subgroup analyses, there was moderate heterogeneity between the studies performed in the Caucasian population and between the studies concerning type 1 and type 2 diabetes. The random effects pooled ORs for the Caucasians and type 2 diabetes were not significant (Table 3). In the studies concerning type 1 diabetes, the pooled OR by fixed and random effects were significant: $\mathrm{OR}=1.29[95 \% \mathrm{CI}(1.06,1.56)]$ and $\mathrm{OR}=1.32[95 \% \mathrm{CI}$ $(1.01,1.71)]$, respectively.

In sensitivity analysis (exclusion of the study with the controls not in Hardy-Weinberg equilibrium), there was no between-study heterogeneity either for the main analysis or for the subgroup analyses (Table 3). Then, the fixed effects ORs were significant and showed an association in the main analysis $(\mathrm{OR}=1.34[95 \% \mathrm{CI}$ $(1.13,1.60)])$, in Caucasians $(\mathrm{OR}=1.29$ [95\% CI $(1.08$, 1.56)]) and in type 2 diabetes $(\mathrm{OR}=1.69$ [95\% CI (1.09, 2.63)]).

The genotype contrast of the homozygotes derived significant association only for the studies in HardyWeinberg equilibrium (fixed effects OR $=1.70[95 \% \mathrm{CI}$ $(1.15,2.53)]$ and random effects $\mathrm{OR}=1.91[95 \% \mathrm{CI}$ $(1.00,3.46])$. The dominant model for the effect of (1.1) allele produced the same pattern of results with the allele contrast, and the recessive model produced no significant associations. These results indicated that allele (1.1) could be dominant for diabetic nephropathy (Table 3 ).

\section{Potential bias}

None of the studies reported that genotyping was blinded to clinical status. Cumulative meta-analysis and recursive meta-analysis for the allelic contrast showed that random effects pooled OR fluctuated from 1.40 in 1998 (first study) to 1.69 in 1999 (two studies) and then declined to 1.26 in 2002 (six studies). Between-study

Table 2 Distribution of the GLUT1 $X b \alpha$ I genotypes and the frequency of the $X b \alpha \mathrm{I}$ alleles for patients with diabetic nephropathy (DN) and for diabetic patients without nephropathy (controls)

\begin{tabular}{|c|c|c|c|c|c|c|c|c|c|c|c|c|}
\hline \multirow[t]{2}{*}{ First author } & \multirow[t]{2}{*}{ Year } & \multirow[t]{2}{*}{ Racial decent } & \multicolumn{6}{|c|}{ Distribution of GLUT1 $X b \alpha$ I genotypes } & \multicolumn{4}{|c|}{ Frequency of $X b \alpha \mathrm{I}$ alleles } \\
\hline & & & $\mathrm{DN}$ & Control & $\mathrm{DN}$ & Control & $\mathrm{DN}$ & Control & $\mathrm{DN}$ & Control & $\mathrm{DN}$ & Control \\
\hline Gutierrez & 1998 & Caucasian & 6 & 43 & 11 & 45 & 3 & 12 & 23 & 131 & 17 & 69 \\
\hline Liu & 1999 & Asian & 12 & 22 & 48 & 20 & 4 & 3 & 72 & 64 & 56 & 26 \\
\hline Tarnow & 2001 & Caucasian & 72 & 94 & 87 & 74 & 16 & 24 & 231 & 262 & 119 & 122 \\
\hline $\mathrm{Ng}$ & 2002 & Caucasian & 106 & 99 & 106 & 90 & 37 & 18 & 318 & 288 & 180 & 126 \\
\hline
\end{tabular}


Table 3 Summary estimates for the odds ratio (OR) of GLUT1 in various allele/genotype contrasts, the significance level $(P$ value) of heterogeneity test ( $Q$ test), and the $I^{2}$ metric (when negative is set to zero): overall analysis, subgroup analyses, and sensitivity analyses. $H W E$ Hardy-Weinberg equilibrium

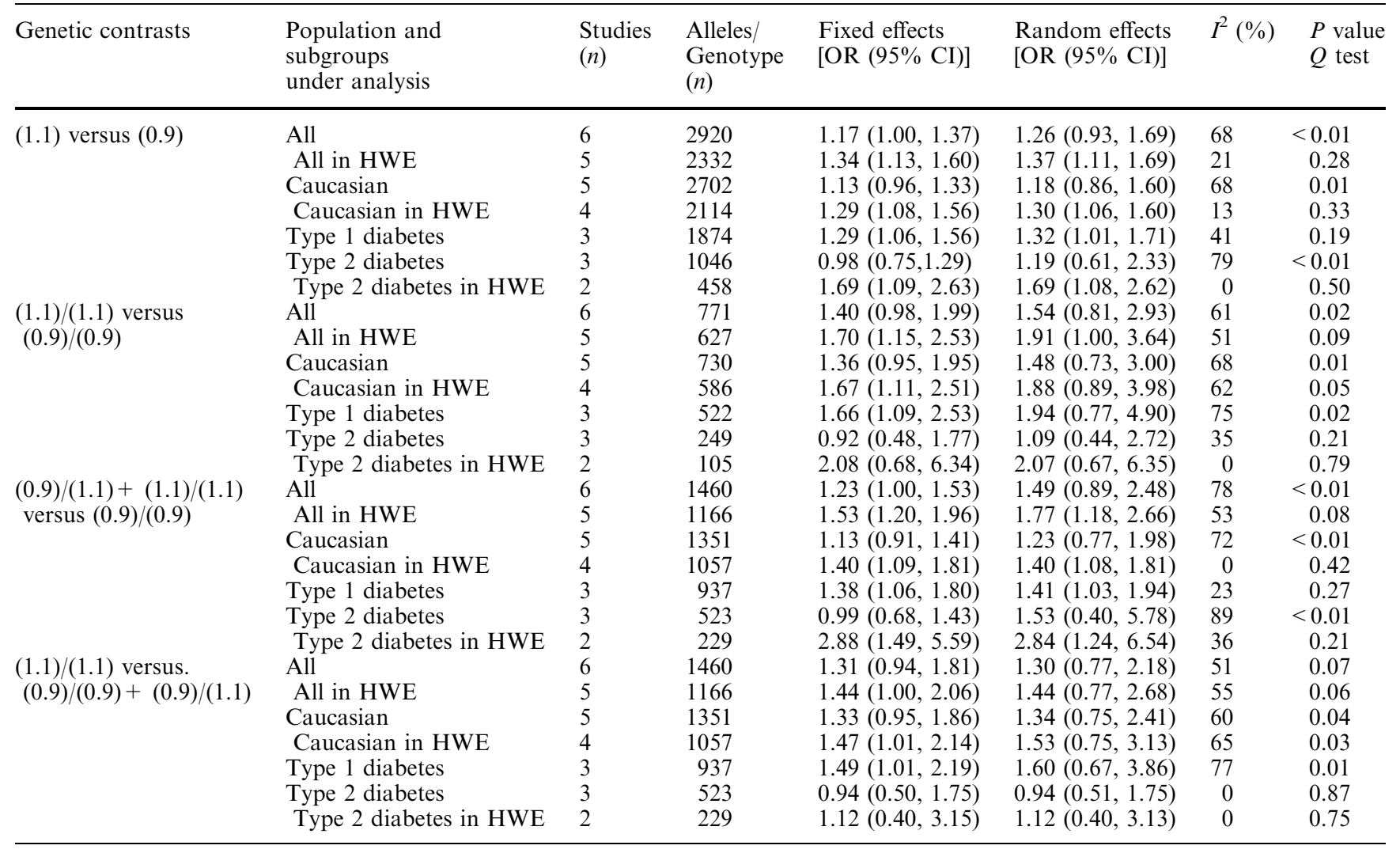

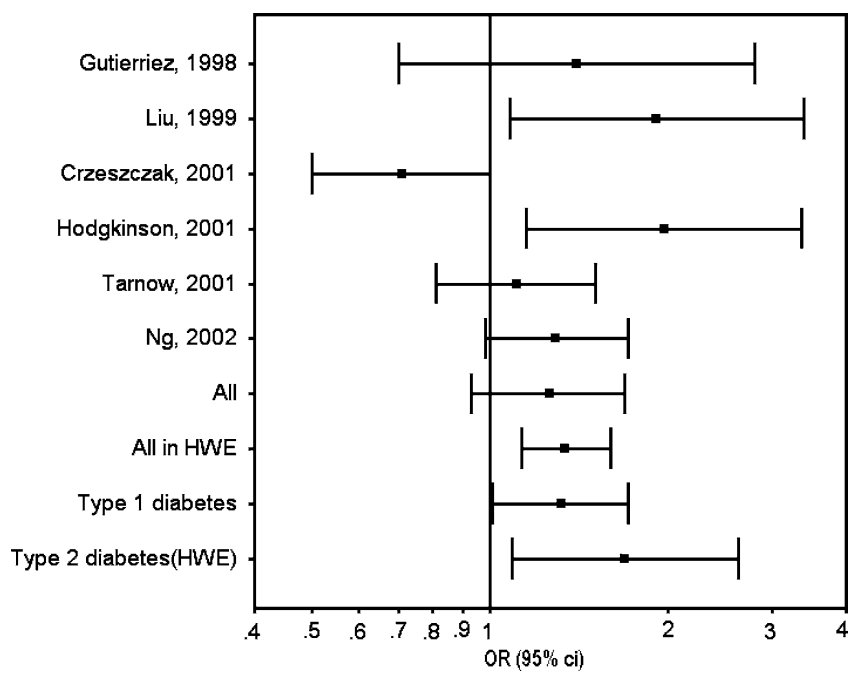

Fig. 1 GLUT1 polymorphism and the risk of diabetic nephropathy: contrast of allele (1.1) against (0.9). Each study is shown by an odds ratio (OR) estimate with the corresponding $95 \%$ confidence interval. For the sensitivity analysis, the fixed effects pooled odds ratio is shown; otherwise, the random effects pooled odds ratio is shown. The horizontal axis is plotted on a log scale

heterogeneity first appeared in $2001\left(P<0.01, I^{2}=73 \%\right)$ and remained in $2002\left(P<0.01, I^{2}=68 \%\right)$ with the last study $(\mathrm{Ng}$ et al. 2002) showing no association. There was also a suggestion that small studies (Liu et al. 1999, Hodgkinson et al. 2001) produced significant association and, in contrast, large studies (Tarnow et al. 2001, Ng et al. 2002) produced no significant associations. The $\alpha$ statistic was significant: $\alpha=2.59$ [95\% CI $(0.04,5.13)$ ], indicating that there was difference between the smaller and larger published studies. However, this result might not be so reliable since the number of studies was relatively small (Ioannidis et al. 2003).

\section{Discussion}

The glucose transporter GLUT1 is the most important representative of the family of facilitative glucose transporters in glomerular mesangial cells. Its expression on the cell surface is probably pivotal in raising intracellular glucose levels in diabetes mellitus (Heilig et al. 1995, 1997). In mesangial cells, elevated intracellular glucose as a result of diabetes mellitus is thought to affect a number of cellular pathways known to be involved in cellular growth and in the accumulation of the extracellular matrix (Larkins et al. 1992; Mahadevan et al. 1995). Exactly these pathological changes are central factors in the pathogenesis of diabetic nephropathy. From this perspective, it becomes clear that the activity of glucose transporter GLUT1 on the cell sur- 
face of the mesangial cells may be rate limiting for the development of the pathological changes in diabetic nephropathy. Cell culture studies have shown that overexpression of GLUT1 in mesangial cells can lead in a matrix accumulation even in the absence of enhanced glucose levels in the medium (Mahadevan et al. 1995; Mogyorosi et al. 1999), indicating that GLUT1 may play a role in the pathogenesis of diabetic nephropathy in both types of diabetes.

This meta-analysis included data from six casecontrol GLUT1 association studies from 710 subjects who developed diabetic nephropathy, along with their respective controls (750 diabetic subjects without nephropathy). These numbers are relative small, and therefore, any inferences have to be cautious (Ioannidis et al. 2003). However, the strength of this analysis is based on the aggregation of published case-control studies, thus better power is achieved to identify an effect of the allele under investigation (Zintzaras and Hadjigeorgiou 2004).

The overall results did not support an association of the GLUT1 polymorphism with diabetic nephropathy and showed a large heterogeneity between study results. This lack of association and the existence of heterogeneity were due to the study with controls not in HardyWeinberg equilibrium. The heterogeneity included racial descent of the study population, types 2 and 1 diabetes, wide range of diagnostic criteria of diabetic nephropathy ranging from proteinuria and/or CRF, different duration of diabetes, demographics including different age and gender distribution, and probably different therapeutic measures used, suggesting the limits of metaanalysis. However, the subgroup analyses revealed an association only for type 1 diabetes. Sensitivity analysis showed that heterogeneity could be explained when data were limited according to Hardy-Weinberg equilibrium. In this case, there was an overall association and association for the Caucasian population and type 2 diabetes.

The two largest studies (Caucasians with type 1 diabetes) failed to show association while only two small studies (Caucasians with type 1 diabetes and Asians with type 2 diabetes) have claimed a susceptibility effect to diabetic nephropathy. These results might be a source of potential publication bias.

Case-control studies of genetic associations have drawbacks, e.g., population structure may cause an allele to be in disequilibrium but not linked to the disease. In addition, case-control studies may have fallen out of favor in studies of association of a gene polymorphism with the risk of a disease because of the possible effect of population stratification, which may not be known. If population substructure affects the gene-disease association, then it should also affect allele frequencies of other genes as well. However, genes that are markers of population substructure and that segregate independently from the investigated gene and are not themselves associated with disease or in linkage disequilibrium with genes associated with disease can be used to make inferences about the existence of substructure in a sample and to consider it in the analysis (Pritchard et al. 1999; Weiss et al. 2001; Satten et al. 2001; Cardon et al. 2003). It is possible that different susceptibility genes are involved in the two types of diabetes (types 1 and 2), which may interact with GLUT1 (Moczulski et al. 1998; Imperatore et al. 1998). The pathogenesis of diabetic nephropathy is complex and includes many inducing factors, and (1.1) allele might be only one of multiple other genetic risk factors for the development of diabetic nephropathy.

Furthermore, the polymorphic $X b \alpha \mathrm{I}$ site is located on the second intron of the GLUT1 gene. As an intronic mutation, the (1.1) allele cannot possibly cause changes in the protein sequence and therefore its functional significance seems questionable. Additionally, it has never been associated to alterations of the GLUT1 expression, even if it is localized in close proximity to the GLUT1 gene enhancer region. A major concern is that $X b \alpha \mathrm{I}$ polymorphism is not the causative polymorphism but is assumed to be in disequilibrium with another locus that has an etiologic role in diabetic nephropathy. The meta-analysis may be subject to error because the difference in the haplotype structure, based on the linkage disequilibrium around $X b \alpha \mathrm{I}$, may be different in study populations. In linkage studies, the GLUT1 region (1p35-p31.3) has not been reported to be an important susceptibility locus in diabetic nephropathy (Imperatore et al. 1998, Moczulski et al. 1998, Vardarli et al. 2002, Lindner et al. 2003, Iyengar et al. 2003).

This meta-analysis was based on unadjusted estimates; a more precise analysis could be performed if adjusted (by gender, age, disease duration) estimates were provided in the studies. The results of a metaanalysis depend on the study design, the inclusion criteria of the cases, and the controls in each study. In this meta-analysis, the cases and controls were well defined with similar inclusion criteria, albeit they unavoidably covered a wide spectrum of disease in terms of duration and other manifestations.

There was some variation in case definition for diabetic nephropathy in the studies. Particularly, the presence of microalbuminuria (urinary albumin secretion $30-300 \mathrm{mg} / 24 \mathrm{~h}$ ) was a diagnostic criterion for diabetic nephropathy in some studies while in others, more advanced stages of nephropathy (including proteinuria and impaired renal function) are considered. These different case definitions are obviously not equivalent.

Variability of the case inclusion criteria is a central possible confounding factor in all studies on the role of genetic markers, such as the GLUT1 gene polymorphism, in diabetic nephropathy. Taking into account the above findings, we defined as cases with diabetic nephropathy only those with proteinuria and/or with CRF. Patients with microalbuminuria were not considered as cases of diabetic nephropathy. These strict selection criteria ensured a clear case definition for our meta-analysis. 
In diabetes type 1 , the absence of microalbuminuria along with a normal renal function practically excludes the actual presence of a diabetic nephropathy. In all studies on diabetes type 1, controls were clearly defined by the absence of microalbuminuria. In two studies on diabetes type 1 , the definition of the controls was absence of microalbuminuria after 15 and 20 years, respectively. In the third study, the duration of diabetes was found to be longer than 13 years. Renal involvement generally begins $10-15$ years after the onset of type 1 diabetes. However, even after 10-15 years of diabetes, the absence of microalbuminuria does not exclude the possibility of developing nephropathy later in life. In an important clinical study, Quinn et al. (1996) demonstrated that the incidence of nephropathy does not plateau until 30 years of type 1 diabetes (Quinn et al. 1996). Patients without albuminuria after 20 years have a yearly risk of developing overt renal disease of about $1 \%$ (Krolewski et al. 1987). This means that the controls in the analyzed studies represented merely part of the patients who are at risk for nephropathy.

In diabetes type 2 , it is more difficult to establish the presence or absence of a diabetic nephropathy, at least using the same criteria as in diabetes type 1. In patients with diabetes type 2 , the specificity of microalbuminuria is lower than in insulin-dependent diabetes patients (type 1). Proteinuria without evidence of diabetic nephropathy may be due to other factors such as hypertension and vascular kidney disease. The above confounding factors can lead to difficulties in the definition of the cases with diabetic nephropathy in diabetes type 2 . This means that any possible conclusion should be interpreted cautiously in studies of genetic markers in this group of patients. Furthermore, the duration of disease cannot be defined with the same accuracy as in type 1 diabetes. Type 2 diabetes begins in most cases subclinically, and the disease duration is much longer than the clinically assessed. In all three diabetes type 2 studies, controls (normoalbuminuric patients) had a disease duration longer than 10 years. This fact makes it unlikely that the nephropathy in type 2 diabetic patients considered in this analysis was due to nondiabetic renal disease.

In conclusion, the meta-analysis and the subsequent sensitivity analyses supported an association between the GLUT1 gene $X b \alpha I$ polymorphism and diabetic nephropathy. This study is interesting and a good example to show how data not in Hardy-Weinberg equilibrium, especially in controls, affect the overall analytical results. The impact of excluding such data was here so big that it led us to obtain the significant findings, indicating the importance of the data quality even in the meta-analysis.

Finally, the conclusion is based on a relatively small number of studies and participants, and any inferences have to be cautious. The investigation of genetic associations requires large population studies, but for many of them, truly large studies with thousands of participants might never be conducted (Ioannidis et al. 2003). Therefore, every relevant information should be first examined thoroughly and then be incorporated into a meta-analysis.

Acknowledgements We thank Thomas Trikalinos for comments and discussion.

\section{References}

The Diabetes Control and Complications Trial Research Group (1993) The effect of intensive treatment of diabetes on the development and progression of long-term complications in insulin-dependent diabetes mellitus. N Engl J Med 329:977-986

S.A.G.E. Statistical analysis for genetic epidemiology. (2003) Computer program package available from Statistical Solutions Ltd, Cork, Ireland

Cardon LR, Palmer LJ (2003) Population stratification and spurious allelic association. Lancet 361:598-604

Cochran WG (1954) The combination of estimates from different experiments. Biometrics 10:101-129

de Jong DJ, van der Logt EM, van Schaik A, Roelofs HM, Peters WH, Naber TH (2003) Genetic polymorphisms in biotransformation enzymes in Crohn's disease: association with microsomal epoxide hydrolase. Gut 52:547-551

Egger M, Davey SG, Schneider M, Minder C (1997) Bias in metaanalysis detected by a simple, graphical test. BMJ 315:629-634

Grzeszczak W, Moczulski DK, Zychma M, Zukowska-Szczechowska E, Trautsolt W, Szydlowska I (2001) Role of GLUT1 gene in susceptibility to diabetic nephropathy in type 2 diabetes. Kidney Int 59:631-636

Gutierrez C, Vendrell J, Pastor R, Broch M, Aguilar C, Llor C, Simon I, Richart C (1998) GLUT1 gene polymorphism in noninsulin-dependent diabetes mellitus: genetic susceptibility relationship with cardiovascular risk factors and microangiopathic complications in a Mediterranean population. Diabetes Res Clin Pract 41:113-120

Heilig CW, Concepcion LA, Riser BL, Freytag SO, Zhu M, Cortes P (1995) Overexpression of glucose transporters in rat mesangial cells cultured in a normal glucose milieu mimics the diabetic phenotype. J Clin Invest 96:1802-1814

Heilig CW, Liu Y, England RL, Freytag SO, Gilbert JD, Heilig KO, Zhu M, Concepcion LA, Brosius FC, III (1997) D-glucose stimulates mesangial cell GLUT1 expression and basal and IGF-I-sensitive glucose uptake in rat mesangial cells: implications for diabetic nephropathy. Diabetes 46:1030-1039

Higgins JP, Thompson SG, Deeks JJ, Altman DG (2003) Measuring inconsistency in meta-analyses. BMJ 327:557-560

Hodgkinson AD, Millward BA, Demaine AG (2001) Polymorphisms of the glucose transporter (GLUT1) gene are associated with diabetic nephropathy. Kidney Int 59:985-989

Imperatore G, Hanson RL, Pettitt DJ, Kobes S, Bennett PH, Knowler WC (1998) Sib-pair linkage analysis for susceptibility genes for microvascular complications among Pima Indians with type 2 diabetes. Pima Diabetes Genes Group. Diabetes 47:821-830

Ioannidis JP (1998) Effect of the statistical significance of results on the time to completion and publication of randomized efficacy trials. JAMA 279:281-286

Ioannidis JP, Trikalinos TA, Ntzani EE, Contopoulos-Ioannidis DG (2003) Genetic associations in large versus small studies: an empirical assessment. Lancet 361:567-571

Iyengar SK, Fox KA, Schachere M, Manzoor F, Slaughter ME, Covic AM, Orloff SM, Hayden PS, Olson JM, Schelling JR, Sedor JR (2003) Linkage analysis of candidate loci for endstage renal disease due to diabetic nephropathy. J Am Soc Nephrol 14:S195-S201

Krolewski AS, Warram JH, Rand LI, Kahn CR (1987) Epidemiologic approach to the etiology of type I diabetes mellitus and its complications. N Engl J Med 317:1390-1398

Larkins RG, Dunlop ME (1992) The link between hyperglycaemia and diabetic nephropathy. Diabetologia 35:499-504 
Lau J, Antman EM, Jimenez-Silva J, Kupelnick B, Mosteller F, Chalmers TC (1992) Cumulative meta-analysis of therapeutic trials for myocardial infarction. N Engl J Med 327:248-254

Lau J, Ioannidis JP, Schmid CH (1997) Quantitative synthesis in systematic reviews. Ann Intern Med 127:820-826

Lindner TH, Monks D, Wanner C, Berger M (2003) Genetic aspects of diabetic nephropathy. Kidney Int 63 (Suppl 84):S186S191

Liu ZH, Guan TJ, Chen ZH, Li LS (1999) Glucose transporter (GLUT1) allele (XbaI-) associated with nephropathy in noninsulin-dependent diabetes mellitus. Kidney Int 55:1843-1848

Mahadevan P, Larkins RG, Fraser JR, Fosang AJ, Dunlop ME (1995) Increased hyaluronan production in the glomeruli from diabetic rats: a link between glucose-induced prostaglandin production and reduced sulphated proteoglycan. Diabetologia 38:298-305

Moczulski DK, Rogus JJ, Antonellis A, Warram JH, Krolewski AS (1998) Major susceptibility locus for nephropathy in type 1 diabetes on chromosome 3q: results of novel discordant sib-pair analysis. Diabetes 47:1164-1169

Mogyorosi A, Ziyadeh FN (1999) GLUT1 and TGF-beta: the link between hyperglycaemia and diabetic nephropathy. Nephrol Dial Transplant 14:2827-2829

Ng DP, Canani L, Araki S, Smiles A, Moczulski D, Warram JH, Krolewski AS (2002) Minor effect of GLUT1 polymorphisms on susceptibility to diabetic nephropathy in type 1 diabetes. Diabetes 51:2264-2269

Ntais C, Polycarpou A, Ioannidis JP (2003a) Association of the CYP17 gene polymorphism with the risk of prostate cancer: a meta-analysis. Cancer Epidemiol Biomarkers Prev 12:120-126

Ntais C, Polycarpou A, Ioannidis JP (2003b) SRD5A2 gene polymorphisms and the risk of prostate cancer: a meta-analysis. Cancer Epidemiol Biomarkers Prev 12:618-624

Pritchard JK, Rosenberg NA (1999) Use of unlinked genetic markers to detect population stratification in association studies. Am J Hum Genet 65:220-228

Quinn M, Angelico MC, Warram JH, Krolewski AS (1996) Familial factors determine the development of diabetic nephropathy in patients with IDDM. Diabetologia 39:940-945

Satten GA, Flanders WD, Yang Q (2001) Accounting for unmeasured population substructure in case-control studies of genetic association using a novel latent-class model. Am J Hum Genet $68: 466-477$
Silverman EK, Palmer LJ (2000) Case-control association studies for the genetics of complex respiratory diseases. Am J Respir Cell Mol Biol 22:645-648

Strojek K, Grzeszczak W, Morawin E, Adamski M, Lacka B, Rudzki H, Schmidt S, Keller C, Ritz E (1997) Nephropathy of type II diabetes: evidence for hereditary factors? Kidney Int 51:1602-1607

Tarnow L, Grarup N, Hansen T, Parving HH, Pedersen O (2001) Diabetic microvascular complications are not associated with two polymorphisms in the GLUT-1 and PC-1 genes regulating glucose metabolism in Caucasian type 1 diabetic patients. Nephrol Dial Transplant 16:1653-1656

Valderrábano F, Berthoux FC, Jones EH, Mehls O (1996) Report on management of renal failure in Europe, XXV, 1994 end stage renal disease and dialysis report. The EDTA-ERA Registry. European Dialysis and Transplant Association- European Renal Association. Nephrol Dial Transplant 11(Suppl 1):S2S21

Vardarli I, Baier LJ, Hanson RL, Akkoyun I, Fischer C, Rohmeiss P, Basci A, Bartram CR, Van Der Woude FJ, Janssen B (2002) Gene for susceptibility to diabetic nephropathy in type 2 diabetes maps to 18q22.3-23. Kidney Int 62:2176-2183

Weir BS (1996) Genetic data analysis II: Methods for discrete population genetic data. Sinauer Associates, Sunderland MA, USA

Weiss ST, Silverman EK, Palmer LJ (2001) Case-control association studies in pharmacogenetics. Pharmacogenomics J 1:157158

Whitehead A (1997) A prospectively planned cumulative metaanalysis applied to a series of concurrent clinical trials. Stat Med 16:2901-2913

Whitehead A (2002) Meta-analysis of controlled clinical trials. Wiley, Chichester

Xu J, Turner A, Little J, Bleecker ER, Meyers DA (2002) Positive results in association studies are associated with departure from Hardy-Weinberg equilibrium: hint for genotyping error? Hum Genet 111:573-574

Zintzaras E, Hadjigeorgiou GM (2004) Association of paraoxonase 1 (PON1) gene polymorphisms with risk of Parkinson's disease: a meta-analysis. J Hum Genet 49:474-481

Zintzaras E, Ioannidis JP (2004) Heterogeneity testing in metaanalysis of genome searches. Genet Epidem 28:123-137 